\title{
Composición corporal, somatotipo, rendimiento en salto vertical y consumo máximo de oxígeno en futbolistas profesionales y universitarios
}

\author{
Body composition, somatotype, vertical jump performance and maximal \\ oxygen consumption in professional and university soccer players
}

\section{*Iván Ignacio Molina-Márquez, ${ }^{* *}$ Nicolás Gómez-Álvarez, ${ }^{* * *}$ Claudio Hernández-Mosqueira \& ****Gustavo Pavez-Adasme}

Molina, I., Gómez, N., Hernández, C., \& Pavez, G. (2021). Composición corporal, somatotipo, rendimiento en salto vertical y consumo máximo de oxígeno en futbolistas profesionales y universitarios Revista Ciencias de la Actividad Física UCM, 22(2), julio-diciembre, 44-56. http://doi.org/10.29035/rcaf.22.2.4

\section{RESUMEN}

Objetivo: El objetivo de este estudio fue describir y comparar la composición corporal, somatotipo, rendimiento en el salto vertical y consumo máximo de oxígeno en futbolistas profesionales y universitarios de Chillán.

Metodología: El presente estudio es de tipo transversal, descriptivo y comparativo, con una muestra no probabilística y por conveniencia. Se evaluaron a 52 futbolistas varones; 18 corresponden a jugadores profesionales y 34 universitarios. El perfil antropométrico (índice de masa corporal, composición corporal y somatotipo) se obtuvo a través de los protocolos de la Sociedad Internacional para el Avance de la Kinantropometría (ISAK), el consumo máximo de oxígeno (VO2 máx) por medio del Yo-Yo test IR1 y capacidad de salto a través del protocolo de Bosco, utilizando una plataforma de contacto Axon Jump. El análisis estadístico se realizó con el programa SPSS V.21; para comparar a ambos grupos se utilizó el estadístico T- Student para muestras independientes $(p<0,05)$.

Resultados: Los resultados muestran que los futbolistas profesionales reportan un mayor porcentaje de masa muscular ( $p<0,001)$ y un menor porcentaje de masa grasa $(p<0,001)$ en comparación a los universitarios. Los análisis de somatotipo indican que los jugadores profesionales se clasifican como meso-ectomórfico en comparación a los jugadores universitarios, quienes se clasificaron como meso-endomórfico. Finalmente, en cuanto a los resultados en las pruebas físicas los profesionales obtuvieron diferencias significativas en la capacidad de salto $(p<0,001)$ y en el VO $\mathrm{O}_{2}$ máx ( $\left.p<0,001\right)$.

Conclusión: Los futbolistas profesionales tienen características corporales, somatotípicas y físicas que les permiten sobresalir con respecto a los futbolistas universitarios. Estos datos servirán a los entrenadores universitarios para disponer de los parámetros respecto de características y condiciones a trabajar para acercarse al alto rendimiento.

Palabras clave: Deporte de equipo, Test de Bosco, Yo-Yo test IRT, selección de talento.

\footnotetext{
* Magíster en Ciencias de la Salud y el deporte/ Magíster Ciencias Biológicas. Centro de Formación Técnico Santo Tomas. Chillán, Chile.

ivan_molinam@hotmail.com | https://orcid.org/0000-0001-6003-5107

** Magíster en Medicina y Ciencias del Deporte. Universidad Adventista de Chile, Chillán, Chile.

nicolasgomez@unach.cl |https://orcid.org/0000-0003-3987-4189

*** Doctor en Ciencias de la Motricidad Humana. Universidad de La Frontera, Temuco, Chile. claudiomarcelo.hernandez@ufrontera.cl | https://orcid.org/0000-0001-9392-2319

**** Magíster en Educación en Salud y Bienestar Humano. Universidad Adventista de Chile. Chillán, Chile. gustavopavez@unach.cl | https://orcid.org/0000-0003-4377-384X
} 


\section{ABSTRACT}

Objective: The objective of this study was to describe and compare the body composition, somatotype, vertical jump performance and maximum oxygen consumption in professional and university soccer players from Chillán.

Methodology: The present study is cross-sectional, descriptive and comparative, with a non-probabilistic sample and for convenience. 52 male soccer players were evaluated; 18 correspond to professional players and 34 university players. The anthropometric profile (body mass index, body composition and somatotype) was obtained through the International Society for the Advancement of Kinantropometry (ISAK) protocols, the maximum oxygen consumption (VO2 max) by means of the Yo-Yo test IR1 and ability to jump through the Bosco protocol using an Axon Jump contact platform. Statistical analysis was performed with the statistical program SPSS V.21, to compare both groups the T-Student statistic was used for independent samples $(p<0.05)$.

Results: The results show that professional soccer players report a higher percentage of muscle mass $(p<0.001)$ and a lower percentage of fat mass ( $p<0.001$ ) compared to university players. Somatotype analyzes indicate that professional gamers are classified as meso-ectomorphic compared to college players, who are classified as mesoendomorphic. Finally, regarding the results in the physical tests, the professionals obtained significant differences in jumping ability $(p<0.001)$ and in VO2 $\max (p<0.001)$.

Conclusion: Professional soccer players have bodily, somatotypic and physical characteristics that allow them to stand out comparated to university soccer players. These data will serve university coaches to have the parameters regarding characteristics and conditions to work to approach high performance.

Key words: Team sport, Bosco test, Yo-Yo test IRT, Talent selection

\section{INTRODUCCIÓN}

El fútbol es el deporte más popular del mundo siendo practicado por hombres, mujeres y niños con diferentes niveles de experiencia, con objetivos recreativos o competitivos como el fútbol amateur, universitario o profesional (Stølen et al., 2005). El fútbol está caracterizado por acciones acíclicas e intermitentes donde se requiere cubrir el campo de juego, tanto en el ataque como en defensa (Bangsbo et al., 2006); adicionalmente, su práctica está asociada a varios factores como el psicológico, el planteamiento técnico-táctico dentro del campo de juego y la capacidad física y fisiológica de los jugadores (Manna et al., 2010).

Los futbolistas deben cumplir con altas demandas físicas y fisiológicas durante los enfrentamientos y entrenamientos. Las bioenergéticas, están asociadas primordialmente a sustratos energéticos del tipo fosfágeno y glúcidos mediante la glicólisis anaeróbica y la fosfoliración oxidativa (Kubayi et al., 2017). La vía anaeróbica es de suma importancia, ya que se requiere para recorrer distancias cortas que se ejecutan de forma explosiva como en los cambios de ritmo, giros, patadas y sprint lineal (Krustrup et al., 2006). Por otra parte, la vía aeróbica es esencial, ya que ayuda a los jugadores de fútbol a mantener acciones repetitivas de alta intensidad, 80-90\% de la frecuencia cardíaca máxima (FC máx), y un 70-80\% del consumo máximo de oxígeno ( $\mathrm{VO}_{2}$ máx) (Bujnovky et al., 2019).

La kineantropometría es una disciplina que estudia el cuerpo humano aplicando diversas perspectivas morfológicas. Los factores morfológicos estiman el rendimiento deportivo y vienen determinados por una elevada carga genética, además de la salud, dieta y entrenamiento del sujeto (Lizana et al., 2015). Su aplicación al fútbol tiene como objetivo entender el proceso de rendimiento deportivo y nutrición, además de otras utilidades como la detección de talento y comparación de los somatotipos con el ideal (Almagia et al., 2015). Adicionalmente, se reconoce que las características morfológicas podrían influir 
en las variables físicas y fisiológicas del deportista (Henríquez et al., 2013).

Por lo tanto, valorar estas cualidades podrían colaborar en el desarrollo deportivo en cuanto a la dirección del entrenamiento para llegar a niveles de alto rendimiento por parte de los jugadores universitarios. Si bien, ninguna habilidad, como la técnica y táctica o características físicas y fisiológicas, se pueden utilizar de forma aislada para identificar éxito en el fútbol, evaluaciones de campo validadas se han relacionado positivamente con el rendimiento físico en la realidad del juego, permitiendo discriminar qué jugadores pueden ser etiquetados como sub elite y elite (Bonney et al., 2020); dentro de estas evaluaciones se encuentran por ejemplo: evaluaciones antropométricas que entregan información de la composición corporal y clasificación somatotípica; el salto vertical, el cual evalúa la fuerza y potencia del tren inferior (Bosco et al., 1983) y la prueba de Yo-Yo Test de recuperación intermitente nivel 1 (Yo-YoIR1), que tiene como objetivo medir el consumo máximo de oxígeno (Krustrup et al., 2003).

La evaluación del desempeño deportivo en diferentes niveles de exigencia y categorías, se ha convertido en un componente invaluable para obtener parámetros físicos y fisiológicos que contribuyan a los programas del desarrollo físico de futbolistas con distinto nivel competitivo (Drust et al., 2007). Por lo tanto, el objetivo de este estudio fue describir y comparar la composición corporal, somatotipo, rendimiento en el salto vertical y consumo máximo de oxígeno en futbolistas profesionales y universitarios de Chillán.

\section{MATERIALES Y MÉTODO}

El presente estudio es de tipo transversal, descriptivo y comparativo, con un muestreo no probabilístico y por conveniencia en el que participaron un total de 52 futbolistas varones, de los cuales 18 son profesionales, pertenecientes al plantel de honor del club deportivo Ñublense de segunda división de la liga chilena, y 34 jugadores universitarios que corresponden a diferentes casas de estudios de la ciudad de Chillán.

Todos los participantes debieron cumplir con los siguientes criterios de inclusión y exclusión según fuesen jugadores profesionales o universitarios: Criterios de inclusión para futbolistas universitarios: (1) Ser del sexo masculino; (2) Ser seleccionado universitario; (3) Tener dos años de experiencia en campeonatos comunales, regionales - nacionales. Criterios inclusión futbolistas profesionales: (1) Ser parte del plantel de honor de fútbol masculino de Club Deportivo Ñublense. Los criterios de exclusión para ambos grupos fueron: (1) Presentar alguna lesión o problema médico de cualquier índole. (2) Haber realizado ejercicio intenso el día anterior.

El presente estudio recibió la autorización del comité de ética de la Universidad Adventista de Chile y los autores declararon que los procedimientos seguidos se conformaron a las normas éticas del comité de experimentación humana responsable y de acuerdo con la Asociación Médica Mundial y la Declaración de Helsinki. Todos los evaluados accedieron voluntariamente firmando un consentimiento informado con previa explicación de los potenciales riesgos y beneficios de su participación.

\section{Procedimientos}

Todas las evaluaciones se realizaron durante la jornada de la mañana a partir de las 9:00 AM en tres jornadas. El primer día se evaluó la composición corporal; durante el segundo y tercer día se realizaron las evaluaciones físicas de capacidad de salto y consumo máximo de oxígeno mediante la prueba Yo-Yo test IR1. Cabe señalar que antes de realizar las evaluaciones físicas se aplicó el calentamiento 11+ de la FiFa de 20 minutos, que consiste en carreras de 10-20 metros, multisaltos, trabajo pliométrico y elongación dinámica y estática (Baeza et al., 2017). 


\section{Composición corporal}

La evaluación antropométrica se realizó desde las 9:00 AM hasta las 11:30 AM, siguiendo las directrices del Manual Internacional de Estandarización Antropométrica editado por la ISAK (Hernández et al., 2021). Se midió talla, masa corporal, cuatro pliegues cutáneos (tríceps, subescapular, supraespinal y pierna), dos diámetros óseos (biepicondilar húmero y fémur) y dos perímetros musculares (brazo flexionado en tensión y pantorrilla). La talla y la masa corporal se obtuvieron con un estadiómetro y una balanza digital Tanita ${ }^{\circledR}$ (Tokio, Japón), también se pudo calcular el índice de masa corporal (IMC) dividiendo el peso corporal por la estatura en metros al cuadrado (masa corporal $(\mathrm{kg})$ /estatura $\left.\left(\mathrm{m}^{2}\right)\right)$. La evaluación fue realizada por un antropometrista certificado por ISAK nivel III, junto con un asistente certificado por ISAK nivel 1, cuyo error técnico de medición (intra e interevaluador) está dentro de los límites recomendados por ISAK (<5\% en pliegues cutáneos $y<1 \%$ para el resto de las mediciones). Para las evaluaciones se utilizó un Kit Gaucho Pro "Mercosur", fabricado en Argentina bajo licencia de Rosscraft, Canadá; para su comercialización en el continente americano. Para la determinación de la composición corporal, se consideró el tejido adiposo, muscular, óseo, residual y de piel, utilizando las ecuaciones de González et al. (2019). Además, se estimó la masa muscular por segmentos de los miembros superiores e inferiores con la propuesta hecha por Rodríguez et al. (2012).

\section{Perfil somatotípico}

Los datos de los futbolistas profesionales como universitarios fueron ingresados en el software Somatotype Calculation and Analysis versión 1.1 (San Diego, CA, USA), donde se procesaron para obtener el somatotipo.

Las ecuaciones empleadas para la determinación de cada uno de los componentes del somatotipo fueron (Rodríguez et al., 2014):

\section{Endomorfía}

$$
0,7182+0,1451 * X+0,00068 * X^{2}+0,0000014 * X^{3}
$$

X (es la suma de los pliegues tríceps, subescapular y supra-espinal multiplicado por 170,18/talla del sujeto (cm).

\section{Mesomorfía}

0,585 X diámetro biepicondíleo del húmero + 0,601 X diámetro biepicondíleo del fémur $+0,188$ X perímetro del brazo corregido $+0,161 \times$ perímetro de la pierna corregido - 0,131 X estatura + 4,5

\section{Ectomorfía}

La ectomorfia es el resultado de diferentes ecuaciones en función del índice ponderal recíproco:

Índice Ponderal $=$ talla $(\mathrm{cm}) /$ raíz cúbica del peso $(\mathrm{kg})$.

Tras obtener el índice ponderal se aplicaron las siguientes ecuaciones en función del resultado:

$$
\begin{aligned}
& \text { Si IP } \geq 40,75 \text { Ectomorfia }=(\text { IP. 0,732) }-28,58 \\
& \text { Si } 38,25<I P<40,75 \text { Ectomorfia = (IP. 0,463) - }
\end{aligned}
$$
17,63

Si IP $\leq 38,25$ Ectomorfia $=0,1$

\section{Evaluación salto vertical}

Para los saltos verticales se trabajó a través del protocolo de Bosco (Bosco et al., 1983), con lo que se evaluó salto vertical Squat Jump (SJ), salto con contramovimiento (CMJ) y Abalakov (Bazán et al., 2016) sobre una plataforma de salto contacto Axon Jump, España. Por cada tipo de salto se realizaron tres intentos con un minuto de descanso cada vez, tomándose como resultado el mejor de ellos.

- SJ: Consiste en la realización de un salto partiendo de una flexión de rodillas de $90^{\circ}$, evitando un contramovimiento con el fin de que no se acumule energía elástica. El tronco debe estar recto y las manos situadas en las caderas durante la ejecución de la prueba, evitando que estas se separen del cuerpo. En la fase de vuelo, el sujeto debe mantener 
el cuerpo erguido, piernas extendidas y pies en flexión plantar, efectuando la caída en el mismo lugar de inicio.

- Salto con contramovimiento (CMJ): El evaluado se coloca de pie sobre la plataforma de contacto, con las manos en la cintura, los pies alineados con los hombros y en completo contacto con la plataforma, el tronco erguido y las rodillas en extensión completa. Al estímulo sonoro pronunciado por el evaluador, el individuo realiza un salto, que busca alcanzar su altura máxima (Martins et al., 2018).

- Salto Abalakov: El deportista parte desde una posición erecta; debe tener las manos y brazos libres para ser utilizadas de forma coordinada y sincronizada con la acción de flexo-extensión de las piernas.

\section{Evaluación de consumo máximo de oxígeno relativo y absoluto}

El Yo-YolR1 consiste en una prueba progresiva y máxima, con trayectos de ida y vuelta de 20 metros (40 metros en total), en la que los sujetos corren entre dos líneas marcadas en el suelo (Deprez et al., 2015). Los deportistas tenían que hacer coincidir el sonido emitido por un aparato programado en consonancia con los tiempos establecidos para este nivel (Yo Yo Compact Disc: Helle Thompson, Copenhagen, Denmark) con el momento en que se pisa la línea, habiendo un descanso de 10 segundos entre un desplazamiento y otro. Los sonidos están programados para realizar el primer periodo a una velocidad de $10 \mathrm{~km} / \mathrm{h}$, realizando un incremento progresivo de esta; la prueba se consideró finalizada cuando el individuo no conseguía llegar a la línea de los 20 metros dos veces consecutivas.

Se utilizó la fórmula para el consumo máximo de oxígeno relativo $\left(\mathrm{VO}_{2}\right.$ máx $\left.\mathrm{ml} / \mathrm{kg} / \mathrm{min}\right)=$ metros recorridos * 0,0084 + 34,4
El consumo máximo de oxígeno absoluto se obtuvo multiplicando la masa corporal del sujeto por el $\mathrm{VO}_{2}$ máx relativo (Krustrup et al., 2003)

\section{ANÁLISIS ESTADÍSTICO}

Estadística descriptiva para las variables son presentadas como media \pm desviación estándar. Se evalúo la composición corporal, perfil somatotípico, capacidad de salto (CMJ, SJ y Abalakov) y consumo máximo de oxígeno, en futbolistas profesionales y amateur. La normalidad y homogeneidad de varianza de los datos fueron evaluadas con las pruebas de Kolmogorov Smirnov y Levene, respectivamente. Al distribuirse los datos en forma normal, se empleó la prueba $T$ de student para muestras independientes con el fin de evaluar posibles diferencias entre ambos grupos. Adicionalmente, el Tamaño del Efecto (TE) se calculó con la d de Cohen, considerando un efecto pequeño $(0,2)$, moderado $(0,5)$ o fuerte $(0,8)$ (Sullivan \& Feinn, 2012).Los datos fueron analizados en el paquete estadístico SPSS versión 21.0 (SPSS 21.0 para Windows, SPSS Inc., IL, USA). Para todos Ios análisis se empleó un nivel de alfa de $p<0,05$.

\section{RESULTADOS}

En la Tabla 1, se describen las características generales y antropométricas de los futbolistas profesionales y universitarios. La comparación entre grupos mediante la prueba $T$ - Student para muestras independientes revelaron diferencias significativas en medidas antropométricas básicas (masa corporal; $p<0,001, T E=0,44$ ), diámetros (diámetro humeral biepicondilar; $p<0,001, T E=0,68$; moderado), perímetros (antebrazo máximo; $p<$ 0,005, TE= 0,51; cadera; $p<0,005$, TE= 0,58; muslo máximo; $p<0,05$, TE=0,18; muslo medial; $p<0,05$, TE= 0,62; pantorrilla $p<0,05, \mathrm{TE}=0,25$ ) y pliegues (muslo medial $p<0,05, T E=0,57$ ). 
Tabla 1

Características generales y antropométricas de la muestra según futbolistas profesionales y universitarios.

\begin{tabular}{|c|c|c|c|c|c|}
\hline \multirow{2}{*}{$\begin{array}{l}\text { Variables } \\
\text { Medidas básicas }\end{array}$} & \multirow[t]{2}{*}{$\begin{array}{c}\text { Profesionales } \\
\text { (Media } \pm D E)\end{array}$} & \multirow[t]{2}{*}{$\begin{array}{l}\text { Universitarios } \\
\text { (Media } \pm \text { DE) }\end{array}$} & \multirow[t]{2}{*}{ Valor $p$} & \multicolumn{2}{|c|}{ Tamaño del efecto } \\
\hline & & & & & \\
\hline Edad (años) & $25,0 \pm 3,20$ & $22 \pm 2,2$ & 0,0611 & 0,31 & Pequeño \\
\hline Masa (kg) & $70,1 \pm 2,1$ & $74,8 \pm 1,81$ & $0,0002^{*}$ & 0,44 & Pequeño \\
\hline Talla (cm) & $174 \pm 1,05$ & $172 \pm 1,10$ & 0,0512 & 0,24 & Pequeño \\
\hline $\mathrm{IMC}\left(\mathrm{kg} / \mathrm{m}^{2}\right)$ & $23,85 \pm 7,25$ & $25,10 \pm 2,52$ & 0,0522 & 0,33 & Pequeño \\
\hline \multicolumn{6}{|l|}{ Diámetros (cm) } \\
\hline Biacromial & $39,40 \pm 1,20$ & $40,70 \pm 0,10$ & 0,0623 & 0,22 & Pequeño \\
\hline Tórax transverso & $29,89 \pm 0,80$ & $30,28 \pm 2,22$ & 0,0573 & 0,30 & Pequeño \\
\hline Tórax anteroposterior & $18,88 \pm 1,00$ & $20,26 \pm 3,20$ & 0,0523 & 0,35 & Pequeño \\
\hline Bi-iliocrestidio & $27,82 \pm 1,30$ & $25,04 \pm 4,89$ & 0,0575 & 0,31 & Pequeño \\
\hline Humeral (biepicondelar) & $9,63 \pm 4,18$ & $5,91 \pm 5,40$ & $0,0004^{*}$ & 0,68 & Moderado \\
\hline Femoral (biepicondelar) & $9,80 \pm 2,89$ & $8,24 \pm 2,11$ & 0,1334 & 0,32 & Pequeño \\
\hline \multicolumn{6}{|l|}{ Perímetros $(\mathrm{cm})$} \\
\hline Cabeza & $56,4 \pm 7,42$ & $55,02 \pm 1,20$ & 0,1384 & 0,24 & Pequeño \\
\hline Brazo relajado & $31,05 \pm 1,22$ & $28,79 \pm 2,10$ & 0,0642 & 0,37 & Pequeño \\
\hline Brazo Flexionado en Tensión & $32,34 \pm 4,03$ & $30,89 \pm 3,00$ & 0,0784 & 0,31 & Pequeño \\
\hline Antebrazo máximo & $27,03 \pm 0,32$ & $24,70 \pm 0,85$ & $0,0423^{*}$ & 0,51 & Moderado \\
\hline Tórax mesoesternal & $91,60 \pm 5,48$ & $90,80 \pm 4,11$ & 0,2452 & 0,18 & Pequeño \\
\hline Cintura (mínima) & $78,63 \pm 4,20$ & $78,18 \pm 2,00$ & 0,7422 & 0,10 & Pequeño \\
\hline Cadera (máximo) & $96,45 \pm 2,11$ & $91,27 \pm 4,83$ & $0,0007^{*}$ & 0,58 & Moderado \\
\hline Muslo máximo & $57,22 \pm 7,47$ & $55,82 \pm 3,80$ & $0,0247^{*}$ & 0,18 & Pequeño \\
\hline Muslo (medial) & $52,34 \pm 3,73$ & $49,70 \pm 3,70$ & $0,0255^{*}$ & 0,62 & Moderado \\
\hline Pantorrilla (máxima) & $37,70 \pm 1,58$ & $36,07 \pm 2,03$ & $0,3517^{*}$ & 0,25 & Pequeño \\
\hline \multicolumn{6}{|l|}{ Pliegues (mm) } \\
\hline Tríceps & $7,00 \pm 2,20$ & $9,19 \pm 1,32$ & 0,0341 & 0,41 & Pequeño \\
\hline Subescapular & $8,50 \pm 4,93$ & $10,84 \pm 0,92$ & 0,2452 & 0,29 & Pequeño \\
\hline Supraespinal & $6,88 \pm 2,87$ & $8,23 \pm 1,41$ & 0,0842 & 0,32 & Pequeño \\
\hline Abdominal & $13,75 \pm 6,32$ & $11,81 \pm 3,35$ & 0,0772 & 0,35 & Pequeño \\
\hline Muslo Medial & $10,00 \pm 3,25$ & $14,17 \pm 4,82$ & $0,0342^{*}$ & 0,57 & Moderado \\
\hline Pantorrilla (máxima) & $5,05 \pm 1,23$ & $6,09 \pm 0,60$ & 0,1422 & 0,11 & Pequeño \\
\hline
\end{tabular}

$I M C=$ Índice de masa corporal, * Denota diferencias significativas $(p<0,05)$ mediante estadístico T de Student para muestras independientes. 
En la Tabla 2, se describe la composición corporal, donde se encontraron diferencias significativas entre profesionales y universitarios en el porcentaje de masa grasa, muscular y ósea ( $p<$ $0,05)$. Los futbolistas profesionales presentan un menor porcentaje de masa grasa y un mayor porcentaje de masa muscular y óseo con respecto a los universitarios ( $p=0,0001)$. En cuanto a la masa muscular por segmento solo se encontraron diferencias significativas en la masa muscular del miembro inferior (MMEI) ( $p=0,0001)$. Finalmente, en cuanto al somatotipo se encontraron diferencias en mesomorfía y ectormofía. En todas estas variables se encontró un tamaño del efecto moderado a excepción de la ectomorfia, donde el tamaño del efecto es pequeño.

Tabla 2

Promedio y desviación estándar de la composición corporal de la masa, por segmento muscular y somatotipo de los jugadores.

\begin{tabular}{|c|c|c|c|c|}
\hline \multirow{2}{*}{ Variables } & Profesionales & Universitarios & \multirow{2}{*}{ Valor $p$} & \multirow{2}{*}{ Tamaño del efecto } \\
\hline & Media \pm DE & Media \pm DE & & \\
\hline \% Piel kg & $5 \pm 1,82$ & $6 \pm 1,10$ & 0,3772 & 0,22 \\
\hline \% Grasa kg & $21 \pm 1,52$ & $25 \pm 3,2$ & $0,0001^{*}$ & 0,64 \\
\hline \% Muscular kg & $49 \pm 2,70$ & $46 \pm 3,10$ & $0,0007^{*}$ & 0,57 \\
\hline \% Residual kg & $12 \pm 3,10$ & $13 \pm 2,22$ & 0,0662 & 0,28 \\
\hline \% Ósea kg & $13 \pm 2,20$ & $10 \pm 2,31$ & $0,0007^{*}$ & 0,62 \\
\hline kg MMES & $6,79 \pm 0,64$ & $5,81 \pm 0,67$ & 0,0611 & 0,18 \\
\hline kg MMEI & $15,54 \pm 1,74$ & $10,94 \pm 2,50$ & $0,0007^{*}$ & 0,74 \\
\hline Endomorfía & $2,06 \pm 0,64$ & $2,82 \pm 0,55$ & 0,0722 & 0,31 \\
\hline Mesomorfía & $5,14 \pm 0,83$ & $3,21 \pm 1,39$ & $0,0022^{*}$ & 0,55 \\
\hline Ectomorfía & $2,13 \pm 0,58$ & $1,62 \pm 0,87$ & $0,0078^{*}$ & 0,37 \\
\hline Somatotipo & Meso-ectomórficos & Meso-endomórfico & & \\
\hline
\end{tabular}

\%= Porcentaje, $\mathrm{kg}$ = kilógramos, MMES= Masa muscular extremidad superior, MMEI=Masa muscular extremidad inferior *Denota diferencias significativas $(p<0,05)$ mediante estadístico $T$ de Student para muestras independientes.

En la Figura 1, se aprecia la distribución de ambos grupos en la somatocarta, donde los futbolistas profesionales se clasificaron como mesoectomórficos a diferencia de los jugadores universitarios, donde su clasificación es mesoendomórfico. 


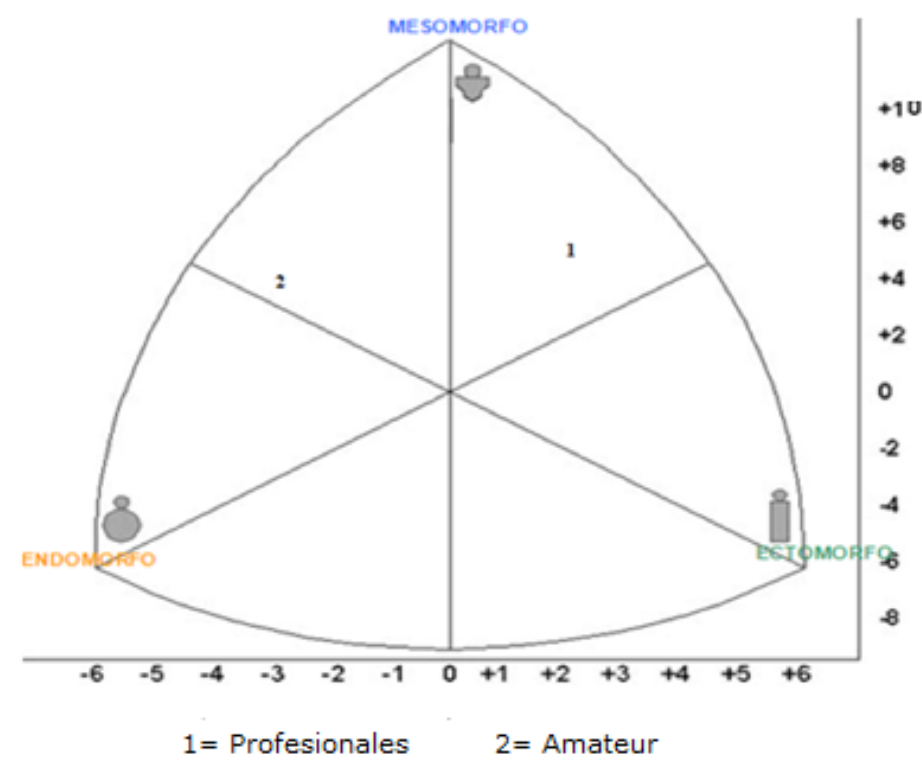

Figura 7. Somatocarta de distribución que indica el somatopunto del promedio de los futbolistas profesionales y universitarios de Chillán.

En la Tabla 3, se encuentran los resultados obtenidos en las pruebas físicas. Los futbolistas profesionales poseen mayor capacidad de salto en los tres saltos evaluados: SJ, Abalakov y CMJ $(p<0,05)$ y mayor capacidad fisiológica (consumo máximo de oxígeno), tanto en el $\mathrm{VO}_{2}$ máx relativo como absoluto ( $p=0,0019)$, donde se evidenció una diferencia significativa. Sin embargo, el tamaño del efecto en la capacidad de salto fue de pequeño a moderado, a diferencia del $\mathrm{VO}_{2}$ máx que fue un efecto moderado.

Tabla 3

Características físicas y fisiológicas de los futbolistas.

\begin{tabular}{|c|c|c|c|c|}
\hline Variables & $\begin{array}{c}\text { Profesionales } \\
\text { media } \pm \text { DE }\end{array}$ & $\begin{array}{c}\text { Universitarios } \\
\text { media } \pm \mathrm{DE}\end{array}$ & $P$ & $\begin{array}{c}\text { Tamaño del } \\
\text { efecto }\end{array}$ \\
\hline \multicolumn{5}{|l|}{ Saltos Verticales } \\
\hline Abalakov (cm) & $48,2 \pm 5,16$ & $38,43 \pm 5,68$ & $0,0001^{*}$ & 0,64 \\
\hline Squat Jump (cm) & $36,28 \pm 5,16$ & $31,21 \pm 4,61$ & $0,0007^{*}$ & 0,34 \\
\hline $\mathrm{CMJ}(\mathrm{cm})$ & $42,64 \pm 4,85$ & $34,63 \pm 4,57$ & $0,0001^{*}$ & 0,52 \\
\hline \multicolumn{5}{|l|}{ Yo-Yo IR1 } \\
\hline $\mathrm{VO}_{2}$ máx relativo (ml/kg/min) & $44,49 \pm 2,62$ & $38,56 \pm 1,91$ & $0,0019 *$ & 0,78 \\
\hline $\mathrm{VO}_{2}$ máx absoluto (L/min) & $3,22 \pm 0,23$ & $2,89 \pm 0,34$ & 0,0019* & 0,74 \\
\hline
\end{tabular}

Yo-Yo $1 R 7=$ Yo-Yo resistencia intermitente nivel 1 *Denota diferencias significativas $(p<0,05)$ mediante estadístico T de Student para muestras independientes.

\section{DISCUSIÓN}

El objetivo de este estudio fue describir y comparar la composición corporal, somatotipo, rendimiento en el salto vertical y consumo máximo de oxígeno en futbolistas profesionales y universitarios de Chillán. Como hallazgos, en el análisis hemos encontrado que los futbolistas profesionales poseen una clasificación de somatotipo meso-ectomorfo en comparación con los universitarios, que se 
clasificaron meso-endomórficos. En cuanto a la composición corporal, se encontró que los futbolistas profesionales poseen mayor masa muscular, masa ósea y menor porcentaje de grasa que los universitarios. Finalmente se detectó que los profesionales superan a los aficionados en el rendimiento del salto vertical y $\mathrm{VO}_{2}$ máx relativo $(p=$ $0,0001)$ y absoluto ( $p=0,0019)$.

La composición corporal es fundamental para el alto rendimiento deportivo de futbolistas con elevado desarrollo muscular y óseo y un bajo contenido graso, con lo que han logrado futbolistas más rápidos, fuertes y potentes y con mejor salud (López et al., 2019). El exceso de tejido adiposo actúa como peso muerto en actividades como correr o saltar. Nuestros resultados, en todo caso, son similares a los reportados en otros futbolistas chilenos (Henríquez et al., 2013), jugadores centroamericanos (González et al., 2019) y europeos (Gligoroska et al., 2015, Sylejmani et al., 2019). Sin embargo, muchas investigaciones han descrito que lo ideal es un somatotipo de mesomorfobalanceado (Henríquez et al., 2013). Paradójicamente, el morfotipo de los futbolistas se asemeja más a atletas de 100 metros planos que a deportes con demandas físicas y fisiológicas similares. Actualmente, esta clasificación ha ido variando principalmente en la liga inglesa, donde está predominado la ectomorfía por sobre la mesomorfía, especialmente en jugadores jóvenes como identificación y desarrollo de talento para mejorar la probabilidad de éxito (Nevill et al., 2019). En cuanto a los futbolistas universitarios, nuestros hallazgos coinciden con otras investigaciones chilenas que reportan que los universitarios nacionales poseen un somatotipo mesoendomórfico, a diferencia de los profesionales, posiblemente debido al tipo de actividad física diaria que efectúan en el día a día, y es que el volumen de entrenamiento físico se ve reducido por las prioridades académicas que limitan las horas de entrenamiento (Almagia et al., 2015).

Al comparar el somatotipo y composición corporal entre futbolistas profesionales $y$ universitarios, se encontró que estos últimos poseen un mayor grado de endomorfía, mayor porcentaje de masa adiposa y un menor grado de mesomorfía y porcentaje de masa muscular con respecto a los profesionales. Sin embargo, estos parámetros morfológicos son altamente modificables con entrenamiento y una dieta adecuada para aumentar principalmente la ecto-mesomorfía independientemente de su nivel y/o posición en el campo de juego (Nevill et al., 2019).

Para evaluar la aptitud física, nuestro estudio consideró el protocolo de Bosco y el Yo-yo test de resistencia intermitente 1. Los saltos verticales incluidos en el protocolo de Bosco tienen como objetivo medir la fuerza, potencia, simetría del tren inferior o capacidad de salto (Hespanhol et al., 2013). Al contrastar los resultados con jugadores noruegos, $54,9 \mathrm{~cm}$ (Wisløff et al., 1998) y colombianos, quienes obtuvieron 39,61 cm (Donado \& Rebolledo, 2020), ambos en el salto CMJ. Por lo que los futbolistas nacionales están por debajo en la capacidad de salto, sin embargo, nuestros hallazgos son similares a los reportados en futbolistas de la serie A italiana (Castagna \& Castellini, 2013). La explicación a las diferencias en el rendimiento en los saltos se debe al porcentaje de grasa corporal. Este porcentaje no tiene en cuenta la masa muscular y se ha reportado que el salto vertical tiene una asociación positiva con un bajo porcentaje de grasa corporal, debido a que desarrolla mayor potencia, por lo tanto, genera mayor velocidad (Abidin \& Adam, 2013). La masa muscular también influye en el salto ya que, a mayor masa muscular existe mayor capacidad de salto, debido al área transversal en músculo y mayor cantidad de proteínas sarcoplasmáticas y contráctiles (Haun et al., 2019), que mayor aceleración, cambio de dirección por parte de los jugadores (Boffi, 2008).

Otro resultado importante arrojado por este estudio fue la diferencia entre profesionales y universitarios en el consumo máximo de oxígeno. Esta diferencia se puede deber a las horas de entrenamiento y al tamaño corporal. El $\mathrm{VO}_{2}$ máx depende del peso, especialmente del peso magro: 
en algunos sujetos se ha encontrado que a mayor masa muscular se evidencian mayores niveles de $\mathrm{VO}_{2}$ máx absoluto (L/min) (Maciejczyk et al., 2014). En consecuencia, el grado de entrenamiento de fuerza, puede inducir aumentos sustanciales en el mismo (Hickson et al., 1980). Por otra parte, al parecer un IMC y porcentaje de grasa corporal aumentado tendrían un efecto negativo en la capacidad cardiorrespiratoria (Cavia et al., 2019). Los valores de $\mathrm{VO}_{2}$ máx reportados en esta investigación están bajo los niveles requeridos para el futbol. Para profesionales oscilan entre 59,2 y 63,2 ml / kg / min, mientras que para aficionados oscila entre 57,8 a 61,7 $\mathrm{ml} /$ kg / min (Ranković et al., 2010).

Estos resultados podrían resultar útiles para comparar la capacidad física y fisiológica de los futbolistas universitarios con respecto a estándares profesionales. Además, permitirá a los especialistas realizar planes de entrenamiento que permitan obtener mejoras en el rendimiento deportivo de sus jugadores

Sin embargo, no son resultados definitivos y este estudio no está libre de limitaciones. La muestra evaluada se limita a una sola región de Chile, por lo que no se puede extrapolar, además, hay que considerar que el equipo profesional pertenece a la serie B de nuestro fútbol chileno, por lo que no puede considerarse como valores de élite. Por otro lado, este estudio no contempló realizar un registro de las horas de entrenamiento, ni conteo de macro y micronutrientes en los evaluados, tampoco consideró el gesto técnico al momento de realizar el salto vertical, especialmente en jugadores universitarios; estas variables hubiesen dado mayor explicación a los resultados obtenidos y podrían constituir las causas de la diferencia de los resultados.

\section{CONCLUSIÓN}

Los resultados de este estudio permiten identificar características diferenciadoras entre futbolistas profesionales y universitarios. Se halló que los futbolistas profesionales poseen una clasificación somatotípicamente meso-ectomorfo, en comparación con los universitarios que se clasificaron meso-endomórficos, además, los profesionales se caracterizaron por una mayor masa muscular, masa ósea y menor porcentaje de grasa. En cuanto al rendimiento físico, en salto vertical y el $\mathrm{VO}_{2}$ máx fue mayor en los futbolistas profesionales que en los universitarios.

El aporte de estos datos puede proporcionar un fundamento científico en la práctica para los entrenadores de fútbol, ya que estos datos pueden tomarse como referencia y parámetros principalmente en el proceso de selección y la conformación de un equipo altamente competitivo y útil, además al momento de direccionar el entrenamiento, especialmente universitario.

\section{CONFLICTO DE INTERÉS}

Los autores declaran no tener ningún conflicto de interés.

\section{REFERENCIAS BIBLIOGRÁFICAS}

Almagia, A., Araneda, A., Sánchez, J., Sánchez, P., Zúñiga, M., \& Plaza, P. (2015). Somatotipo y Composición Corporal de la Selección de Fútbol Masculino Universitario de Chile, Pontificia Universidad Católica de Valparaíso, Campeona los Años 2012 y 2013. International Journal of Morphology, 33(3), 1165-1170. https://doi.org/10.4067/S071795022015000300057

Abidin, N. Z., \& Adam, M. B. (2013). Prediction of Vertical Jump Height from Anthropometric Factors in Male and Female Martial Arts Athletes. The Malaysian Journal of Medical Sciences, 20(1), 39-45. http://www.mjms.usm.my/MJMS20012013/ 05MJMS20012013_oa.pdf

Bangsbo, J., Mohr, M., \& Krustrup, P. (2006). Physical and metabolic demands of training and match-play in the elite football player. Journal of Sports Sciences, 24(7), 665-674. https://doi.org/10.1080/02640410500482529 
Baeza, G., Paredes, G., Vega, P., Monrroy, M., \& Gajardo-Burgos, R. (2017). Effect of "FIFA 11+" on the pattern of fundamental movements in under-14 soccer players. Revista Brasileira de Medicina do Esporte, 23(6), 465-468. https://doi.org/10.1590/1517869220172306173456

Bazán, N., Bruzzese, M., Laiño, F., Ghioldi, M., \& Santa María, C. (2016). Evaluación de la capacidad de salto y estado ponderal en estudiantes de danza clásica de la escuela del Teatro Colón en Buenos Aires. Apunts. Medicina de l'Esport, 57(190), 56-62. https://doi.org/10.1016/j.apunts.2015.07.001

Boffi, F. (2008). Entrenamiento y adaptación muscular: Sustratos y vías metabólicas para la producción de energía. Revista Brasileira de Zootecnia, 37(SPE), 197-201. https://doi.org/10.1590/S151635982008001300022

Bonney, N., Larkin, P., \& Ball, K. (2020). Future Directions and Considerations for Talent Identification in Australian Football. Frontiers in Sports and Active Living, 2, 1-13. https://doi.org/10.3389/fspor.2020.612067

Bosco, C., Luhtanen, P., \& Komi, P. (1983). A simple method for measurement of mechanical power in jumping. European Journal of Applied Physiology and Occupational Physiology, 50(2), 273-282. https://doi.org/10.1007/BF00422166

Bujnovky, D., Maly, T., Ford, K., Sugimoto, D., Kunzmann, E., Hank, M., \& Zahalka, F. (2019). Physical Fitness Characteristics of Highlevel Youth Football Players: Influence of Playing Position. Sports, 7(2), 46. https://doi.org/10.3390/sports7020046

Castagna, C., \& Castellini, E. (2013). Vertical jump performance in Italian male and female national team soccer players. Journal of Strength and Conditioning Research, 27(4), 1156-1161.

https://doi.org/10.1519/JSC.0b013e318261099 9
Cavia, M., Moreno, A., Fernández-Trabanco, B., Carrillo, C., \& Alonso-Torre, S. (2019). Anthropometric characteristics and somatotype of professional soccer players by position. Journal of Sports Medicine and Therapy, 4(4), 73-80. https://doi.org/10.29328/journal.jsmt.100104 7

Deprez, D., Fransen, J., Lenoir, M., Philippaerts, R., \& Vaeyens, R. (2015). The Yo-Yo intermittent recovery test level 1 is reliable in young high-level soccer players. Biology of Sport, 32(1),

65-70. https://pubmed.ncbi.n/m.nih.gov/25729152/

Donado, C., \& Rebolledo-Cobos, R. (2020). Relación entre la capacidad de salto y la estabilidad de la musculatura central en futbolistas profesionales. Revista Cubana de Medicina del Deporte y la Cultura Física, 14(1), e19. http://www.revmedep.sld.cu/index.php/me dep/article/view/19

Drust, B., Atkinson, G., \& Reilly, T. (2007). Future perspectives in the evaluation of the physiological demands of soccer. Sports Medicine, $\quad$ 37(9), 783-805. https://doi.org/10.2165/00007256200737090-00003

Gligoroska, J., Manchevska, S., Efremova, L., Todorovska, L., \& Nikolic, S. (2015). Body composition and maximal oxygen consumption in adult soccer players in the Republic of Macedonia. Journal of Health 5(3), 85-92. https://doi.org/10.17532/jhsci.2016.268

González-Mendoza, R. G., Gaytán-González, A., Jiménez-Alvarado, J. A., Villegas-Balcázar, M., Jáuregui-Ulloa, E. E., Torres-Naranjo, F., \& López-Taylor, J. R. (2019). Accuracy of Anthropometric Equations to Estimate DXA-Derived Skeletal Muscle Mass in Professional Male Soccer Players. Journal of Sports Medicine, 2019, 1-6. https://doi.org/10.1155/2019/4387636 
Haun, C., Vann, C., Roberts, B., Vigotsky, A., Schoenfeld, B., \& Roberts, M. (2019). A Critical Evaluation of the Biological Construct Skeletal Muscle Hypertrophy: Size Matters but So Does the Measurement. Frontiers in Physiology, 10 , 247 https://doi.org/10.3389/fphys.2019.00247

Henríquez-Olguín, C., Báez, E., Ramírez-Campillo, R., \& Cañas, R. (2013). Perfil Somatotípico del Futbolista Profesional Chileno. International Journal of Morphology, 37(1), 225-230. https://doi.org/10.4067/S071795022013000100037

Hernández-Jaña, S., Jorquera-Aguilera, C., AlmagiàFlores, A., Yáñez-Sepúlveda, R., \& RodríguezRodríguez, F. (2021). Composición Corporal y Proporcionalidad en Futbolistas Chilenos. Diferencias entre Categorías Juveniles y Campeones Profesionales. International Journal of Morphology, 39(1), 252-259. https://doi.org/10.4067/s071795022021000100252

Hespanhol, J., de Arruda, M., Cossio, M., \& Silva, R. (2013). Sensitivity and specificity of the strength performance diagnostic by different vertical jump tests in soccer and volleyball at puberty. Revista Brasileira de Medicina Do Esporte, 19(5), 367-370. https://doi.org/10.1590/S151786922013000500014

Hickson, R., Rosenkoetter, M., \& Brown, M. (1980). Strength training effects on aerobic power and short-term endurance. Medicine and Science in Sports and Exercise, 12(5), 336339.

https://pubmed.ncbi.nIm.nih.gov/7453510/
Krustrup, P., Mohr, M., Amstrup, T., Rysgaard, T., Johansen, J., Steensberg, A., Pedersen, P., \& Bangsbo, J. (2003). The yo-yo intermittent recovery test: Physiological response, reliability, and validity. Medicine and Science in Sports and Exercise, 35(4), 697705.

https://doi.org/10.1249/01.MSS.0000058441. 94520.32

Krustrup, P., Mohr, M., Steensberg, A., Bencke, J., Kjær, M., \& Bangsbo, J. (2006). Muscle and Blood Metabolites during a soccer game: Implications for sprint performance. Medicine \& Science in Sports \& Exercise, 38(6),

$1165-1174$. http://doi.org/10.1249/01.mss.0000222845.8 9262.cd

Kubayi, A., Paul, Y., Mahlangu, P., \& Toriola, A. (2017). Physical Performance and Anthropometric Characteristics of Male South African University Soccer Players. Journal of Human Kinetics, 60(1), 153-158. https://doi.org/10.1515/hukin-2017-0098

López, P., Chena, M., Asín, I., Moreno, A., \& Moreno, R. (2019). Efecto de factores contextuales en la composición corporal de jugadores profesionales de fútbol. Un estudio retrospectivo. Nutrición Hospitalaria, 36(6), 1324-1331. https://doi.org/10.20960/nh.02783

Lizana, P., Olivares, R., \& Berral, F. (2015). Somatotype tendency in Chilean adolescents from Valparaíso: Review from 1979 to 2011. Nutrición Hospitalaria, 37(3), 1034-1043. https://doi.org/10.3305/nh.2015.31.3.8425

Maciejczyk, M., Więcek, M., Szymura, J., Szyguła, Z., Wiecha, S., \& Cempla, J. (2014). The Influence of Increased Body Fat or Lean Body Mass on Aerobic Performance. PLOS ONE, 9(4). e95797

https://doi.org/10.1371/journal.pone.0095797 
Manna, I., Khanna, G., \& Chandra, P. (2010). Effect of Training on Physiological and Biochemical Variables of Soccer Players of Different Age Groups. Asian Journal of Sports Medicine, 7(1), 5-22. https://doi.org/10.5812/asjsm.34875

Martins, M., Nunes, E., Rodrigues, C., HernándezMosqueira, C., \& Da Silva, S.. (2018). Características antropométricas y potencia de miembros inferiores en jugadores universitarios de rugby-7. MHSalud: Revista en Ciencias del Movimiento Humano y Salud, 75(2), 1-9. https://doi.org/10.15359/mhs.15-2.4

Nevill, A., Okojie, D., Smith, J., O'Donoghue, P., \& Webb, T. (2019). Are professional footballers becoming lighter and more ectomorphic? Implications for talent identification and development. International Journal of Sports Science \& Coaching, 74(3), 329-335. https://doi.org/10.1177/1747954119837710

Ranković, G., Mutavdžić, V., Toskić, D., Preljević, A., Kocić, M., Nedin-Ranković, G., \& Damjanović, N. (2010). Aerobic capacity as an indicator in different kinds of sports. Bosnian Journal of Basic Medical Sciences, 10(1), 44-48. https://doi.org/10.17305/bjbms.2010.2734

Rodríguez, F., Almagiá, A., \& Berral de la Rosa, F. (2012). Ecuación de Regresión de la Absorciometría de Energía Dual de Rayos $X$ (DEXA) para la Estimación de Segmento de Masa Muscular. International Journal of Morphology, 30(2), 550-556. https://doi.org/10.4067/s071795022012000200031

\section{Dirección para correspondencia}

Iván Ignacio Molina-Márquez

Magíster en Ciencias de la Salud y el deporte/

Magíster Ciencias Biológicas.

Centro de Formación Técnico Santo Tomás.

Chillán, Chile.

ORCID ID: https://orcid.org/0000-0001-6003-5107

Contacto:

ivan_molinam@hotmail.com

Recibido: 07-12-2021

Acentado: 10-06-2021
Rodríguez, X., Castillo, O., Tejo, J., \& Rozowski, J. (2014). Somatotipo de los deportistas de alto rendimiento de Santiago, Chile. Revista Chilena de Nutrición, 47(1), 29-39. https://doi.org/10.4067/s0717-

\section{4}

Stølen, T., Chamari, K., Castagna, C., \& Wisløff, U. (2005). Physiology of soccer: An update. Sports Medicine, 35(6), 501-536. https://doi.org/10.2165/00007256200535060-00004

Sullivan, G., \& Feinn, R. (2012). Using Effect Size-Or Why the $P$ Value Is Not Enough. Journal of Graduate Medical Education, 4(3), 279-282. https://doi.org/10.4300/JGME-D-12-00156.1

Sylejmani, B., Maliqi, A., Gontarev, S., Haziri, S., Morina, B., Durmishaj, E., \& Bajrami, A. (2019). Características Antropométricas y Rendimiento Físico de Jóvenes Futbolistas de Élite de Kosovo. International Journal of Morphology, 37(4), 1429-1436. https://doi.org/10.4067/s071795022019000401429

Wisløff, U., Helgerud, J., \& Hoff, J. (1998). Strength and endurance of elite soccer players. Medicine and Science in Sports and Exercise, 30(3), 462-467. https://doi.org/10.1097/00005768$199803000-00019$ 